\title{
Efectos de la electividad en los cursos de estudio del área sociohumanística, sobre la calidad del aprendizaje en entornos virtuales
}

FECHA DE RECEPCIÓN: 23 de junio FECHA DE APROBACIÓN: 17 de julio Pp. 38-49

Maria Carolina Moreno Salamanca*

Effects an the Selectivity of Sandra Constanza Ortega Ferreira** Saciohumanistic studies courses Adriana Maldonado Currea***

in regard with the Quality of

Learning in Virtual Envianments

Les effets du choix des

matières d'enseignement dans les

cursus d'humanité sur la qualité

de l'apprentissage en

envirunnements virtue/s

Efeitos da eletividade nos cursos

de estudo da área Sócio

Humanística, sobre a qualidade

do aprendizado nos entornos

virtuais

"Magister en Análisis de Problemas Políticos, Económicos e Internacionales Contemporáneos, Universidad

Externado de Colombia en convenio con Ministerio de Relaciones Exteriores, Instituto de Altos Estudios para el Desarrollo y bajo los auspicios del Institut des Haustes Etudes de L'Amérique Latine - Université de Paris III.Profesor Asociado Facultad de Estudios en Ambientes Virtuales Universidad EAN.

*"Doctorando en Psicología con énfasis en neuro-economía, Universidad Nacional de Colombia. Magister en Psicología con énfasis en el estudio de los efectos del estrés sobre el aprendizaje en modelos experimentales, Universidad Nacional de Colombia. .Profesor Asociado Facultad de Estudios en Ambientes Virtuales Universidad EAN.

**** Magister en Enseñanza de la Ciencias Exactas y Naturales, Universidad Nacional de Colombia. .Profesor Asociado Facultad de Estudios en Ambientes Virtuales Universidad EAN. 


\section{RESUMEN}

El presente artículo contiene los resultados de la investigación cuyo objetivo consistía en analizar los efectos de la electividad en los cursos de estudio del área sociohumanística, sobre la calidad del aprendizaje en entornos virtuales con estudiantes de pregrado de la Facultad de Estudios en Ambientes Virtuales de la Universidad EAN. En el estudio se comparó la calidad del aprendizaje en dos grupos expuestos a situaciones experimentales diferentes; en el primero, bajo un modelo de electividad (grupo experimental), y en el segundo, siguiendo un modelo de rigidez (grupo control). Los resultados no arrojan diferencias significativas en la calidad del aprendizaje entre los grupos, pero sí en la percepción de los estudiantes con respecto al tutor y en el nivel de deserción, resultados favorables para el grupo experimental, que presentó una menor deserción y una mejor percepción del tutor.

\section{ABSTRACT}

This paper describes research outcomes whose main obective is to analyze the selectivity of sociohumanistic studies courses regarding the quality of learning in virtual environments of undergraduate students in the School of Virtual Environments at Universidad EAN. In this study, we compared the results obtained out of the observation of two different groups undergoing two different experimental situations; in the first group a model of selectivity was applied (exprimental group) while the second group was exposed to a model of rigidity (control group). The results don't show significant differences in their quality of learning, but there were different perceptions from the students concerning the tutor and the disertion level. In fact, there were better outcomes in the experimental group which experimented lower disertion levels and a better opinion about their tutor.

\section{RESUMÉÉ}

Cet article contient les résultats d'une investigation dont l'objectif réside dans l'analyse des effets $d u$ choix des matières d'enseignement du cursus d'humanité sur la qualité de l'apprentissage en environnements virtuels des étudiants de premier cycle de la Faculté d'Études en Environnements Virtuels de l'Université EAN. Dans cette étude nous avons comparé la qualité de l'apprentissage de deux groupes exposés à des situations expérimentales différentes: le premier groupe est exposé à un modèle de choix (groupe expérimental) et le second groupe se trouve exposé à un modèle de rigidité (groupe control).

Les résultats ne montrent pas de différences significatives sur la qualité de l'apprentissage entre les groupes, mais ils mettent néanmoins en évidence une amélioration de la perception du tuteur de la part des étudiants du groupe expérimental ainsi qu'une diminution du niveau de désertion de ces étudiants.

\section{RESUMO}

O presente artigo contém o resultado de pesquisas científicas cujo objetivo é analisar os efeitos da eletividade nos cursos de estudo da área sócio humanística, sobre a qualidade da aprendizagem em ambientes virtuais com estudantes de graduação da Faculdade de Estudos em Ambientes Virtuais da Universidade EAN. No estudo se comparou a qualidade da aprendizagem em dois grupos expostos a situações experimentais diferentes, o primeiro sob um modelo de eletividade (grupo experimental) e o segundo seguindo um modelo de rigidez (grupo de controle). Os resultados não mostram diferenças significativas na qualidade entre os grupos, porém sim na percepção dos estudantes com respeito ao tutor e no nivel de deserção, resultados favoráveis para o grupo experimental, que apresentou uma menor deserção e uma melhor percepção do tutor.

\section{Palabras clave \\ Ambientes virtuales de aprendizaje, calidad del aprendizaje, deserción estudiantil, electividad, ambiente virtual rígido y ambiente virtual flexible.}

\section{- Key words}

Learning virtual environments, quality of learning, student disertion, selectivity, rigid virtual environment and flexible virtual environment.

\section{$\square$ Motsclefs \\ Plateforme d'apprentissage virtuel, Qualité d'apprentissage, Désertion, choix des matières, \\ Environnement d'apprentissage rigide et Environnement d'apprentissage flexible.}

\footnotetext{
Palavras-chave

Ambientes virtuais de aprendizagem, qualidade da aprendizagem, deserção estudantil, eletividade, ambiente virtual rígido e ambiente virtual flexível.
} 


\section{INTRODUCCIÓN}

$\mathbf{E}^{3}$ ste artículo tiene como objetivo presentar el informe de método y resultados de la segunda fase de la investigación referente a la electividad en los cursos de estudio de negociación intercultural sobre la calidad del aprendizaje en entornos virtuales. En el desarrollo de la investigación se tuvo en cuenta el Modelo Educativo de la Universidad EAN, basado en competencias y que cuenta con un enfoque de currículo investigativo, transversal, pertinente, sistémico y flexible (Universidad EAN, 2013). La Facultad de Estudios en Ambientes Virtuales, fundamenta la creación de los currículos y contenidos virtuales bajo un objetivo social imperante, en palabras de Freire: "la educación es un proceso de transformación social y de diálogos colectivos" (Freire, 2005). El aprendizaje tiene una profunda relación con la transformación (Canudas, 1972) graduada y valiosa de las aptitudes humanas.

El concepto de currículo para esta investigación se basó en el conjunto de elementos para el proceso de enseñanza-aprendizaje y formación, tales como teorías, conceptos, prácticas, metodologías, didácticas, herramientas, y en especial para los ambientes virtuales los contenidos, la interacción y/o comunicación y los aspectos relacionados con el autoaprendizaje (metacognición).

El análisis teórico de la investigación inició con la primera parte del proyecto desarrollado en el año 2013, sobre la Flexibilidad Didáctica

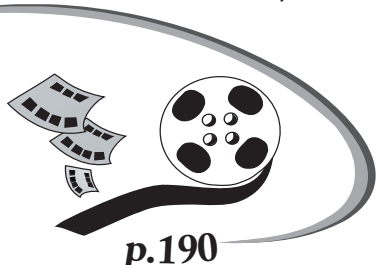

p.190 en Entornos Virtuales de Aprendizaje (Ortega \& Moreno, 2013), que se centró en los enfoques de la teoría educativa crítica de Habermas (citado por Vila, 2011), la teoría psicológica de la enseñanza según Moreira (2000) y el aprendizaje efectivo y significativo, de acuerdo con los planteamientos de Prieto y van de Pol (2006).

El tema de flexibilidad desde la perspectiva del Modelo Educativo de la Universidad EAN, se aborda teniendo en cuenta los siguientes elementos: los créditos académicos, las unidades de estudio nucleares, transversales, anfitriones, electivas, el sistema de ciclos académicos, la no existencia de prerrequisitos en los currículos, la pertinencia, la formación integral y el aspecto sistémico (Universidad EAN, 2013).

La electividad en la Universidad EAN se plantea como un elemento fundamental de la flexibilidad educativa, por ende es parte sinérgica del currículo; según Canudas (1972) el currículo es orgánico y se concibe de modo peculiar en la enseñanza. Para la elaboración de los currículos, plantea Canudas (1972) que lo electivo en la enseñanza es necesario, para reconocer las aptitudes individuales de los estudiantes, ofreciendo diferencias de calidad e intensidad de tipo y jerarquía.

Los currículos electivos permiten la adaptación de la escuela a las diferencias cualitativas de los alumnos, ya que estos pueden optar, dentro de un plan general, entre diversas materias de enseñanza que den libre cauce a sus inclinaciones y aptitudes.

La electividad le plantea al estudiante la posibilidad de multidisciplinariedad y le permite realizar su propia carta de navegación académica según sus intereses, prioridades y campos de profundización de la carrera, ya sean contenidos o ampliación del conocimiento, competencias y habilidades personales, profesionales y organizacionales (Ísola et al., 2013). Es así como la electividad ofrece la posibilidad de incorporar nuevas asignaturas al plan de estudio y 
actividades (Canudas, 1972), durante el periodo de aplicación para el que ha sido redactado. En el caso de la presente investigación y durante la realización de los cursos de negociación intercultural, la electividad se abordó desde la posibilidad de selección de las temáticas y los formatos de presentación de los productos a desarrollar por los estudiantes.

El problema de investigación se planteó de la siguiente manera: ¿Cuáles son los efectos de la electividad en los bloques temáticos en las áreas del conocimiento socio-humanístico, sobre la calidad del aprendizaje en entornos virtuales?
Teniendo en cuenta que se trata de una investigación explicativa, se implementó un diseño experimental donde se manipuló una variable independiente (la electividad) y se observaron sus efectos sobre la variable dependiente (calidad del aprendizaje) en una situación de control. Se requirió el diseño de dos aulas virtuales orientadas a la implementación de los cursos de negociación intercultural para estudiantes del bloque temático Estudios culturales y socio-humanísticos de la Facultad de Estudios en Ambientes Virtuales. Una de las aulas contó con los elementos de electividad en las temáticas y se utilizó un aula tradicional, sin componente electivo para el grupo control.

\section{MÉTODO}

L os participantes en el presente estudio fueron 66, estudiantes de diferentes pro-gramas de pregrado de la Universidad EAN en la modalidad virtual, que se encontraban cursando el bloque temático Estudios culturales y socio-humanísticos y se inscribieron para participar voluntariamente en cursos de negociación intercultural. Los estudiantes fueron inicialmente asignados de manera aleatoria y equitativa a cada grupo, sin embargo, solamente 48 finalizaron el curso: 18 en el grupo control (aula rígida) y 30 en el grupo experimental (aula electiva). La distribución de los grupos fue equitativa por géneros.

El presente estudio tuvo un carácter explicativo (Hernández y Cols, 2010), estableció

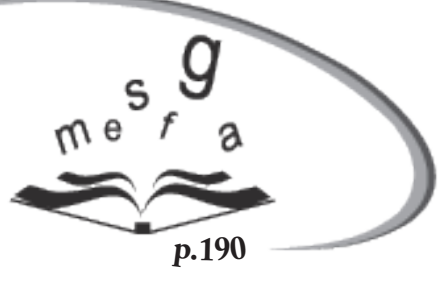

una relación causal entre la electividad (variable independiente) y la calidad del aprendizaje (variable dependiente) en ambientes virtuales.
En este caso, la variable independiente corresponde a la electividad del entorno virtual, entendida como la opción de elegir las temáticas de la unidad de estudios y la manera de presentar las actividades y los productos de las guías de trabajo, con dos grados de manipulación de la variable: presencia-ausencia. Por su parte, la variable dependiente correspondió a la calidad del aprendizaje, entendida como el rendimiento académico, el nivel de competencias desarrolladas, el nivel de deserción estudiantil y la percepción del aprendizaje y el tutor del curso.

El diseño de la investigación fue experimental, puesto que se manipuló una variable independiente y se observaron sus efectos sobre la variable dependiente en una situación de control. En este caso se llevó a cabo un experimento verdadero (se cumple con los criterios de grupos de comparación y equivalencia de los grupos).

La variable dependiente Calidad del aprendizaje, fue medida con base en una serie de indicadores que apuntan a facilitar el análisis 
de cada uno de los elementos implicados en este constructo. Los indicadores fueron: la percepción del aprendizaje, el nivel de desarrollo de las competencias declaradas en el curso, el rendimiento académico en el curso, la evaluación con respecto a la acción tutorial e interacción del tutor en el curso y nivel de satisfacción general en el mismo. Para medir la variable dependiente se diseñó y validó una prueba diagnóstica de evaluación de competencias y un instrumento de medición de satisfacción de los estudiantes con el aula y con los contenidos.

Para efectuar la comparación de resultados obtenidos en cada aula, se utilizó el Software estadístico StatGraphics Plus Centurion, donde se corrieron pruebas $t$ y ANOVA de una vía para todas las variables numéricas. Para el cálculo estadístico de la deserción, se corrió una prueba $Z$ de comparación de proporciones, por tratarse de una variable categórica.

\section{RESULTADOS}

$\mathbf{L}$ os resultados obtenidos de las pruebas de hipótesis, demuestran la existencia o ausencia de diferencias estadísticamente significativas entre los grupos experimental y control en cada una de las variables e indicadores estudiados.

El primer indicador evaluado fue el rendimiento académico, que se calificó sobre 10 puntos y corresponde a la nota obtenida por los estudiantes en el curso. En este caso, el grupo control obtuvo 7.18, mientras que el grupo experimental obtuvo 6.96. La estadística arrojó un puntaje $\mathrm{t}=-0,9993$ y un valor $\mathrm{P}=0,3231$ lo que significa que la inclusión de la electividad no generó efectos sobre el rendimiento académico de los estudiantes (Figura 1).

Figura 1. Comparación del rendimiento académico en los grupos control (rígido) y experimental (electivo).

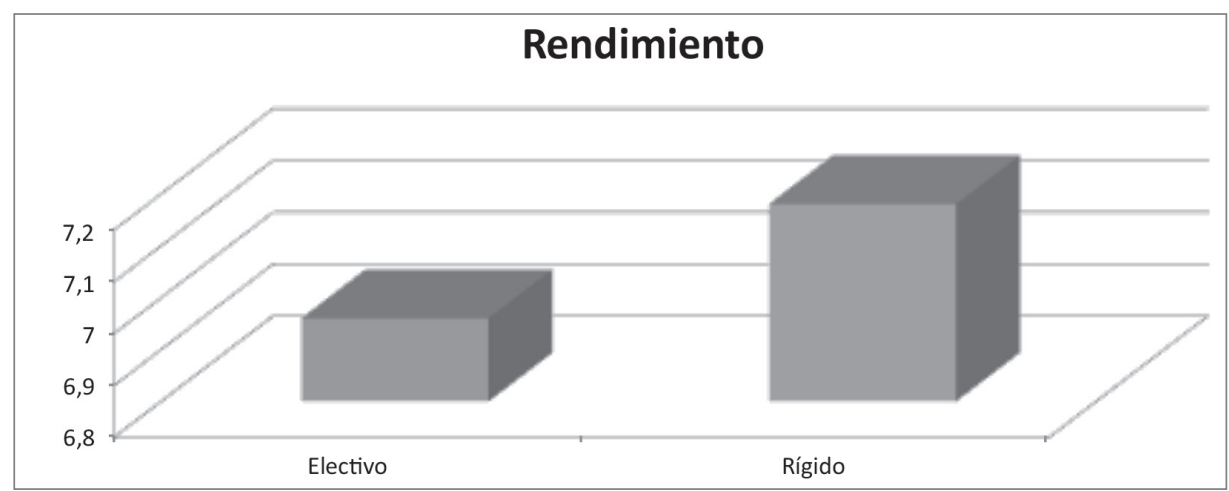

Fuente. Elaboración propia de las autoras.

Los indicadores relacionados con la metodología, tampoco arrojaron diferencias estadísticamente significativas entre los grupos. En este caso el valor fue: $\mathrm{t}=0,566352$ y un $\mathrm{P}=0,578555$ a partir de estos datos se acepta la hipótesis nula, de ausencia de diferencias significativas entre los grupos (Figura 2). 
Figura 2. Comparación la metodología en los grupos control (rígido) y experimental (electivo).

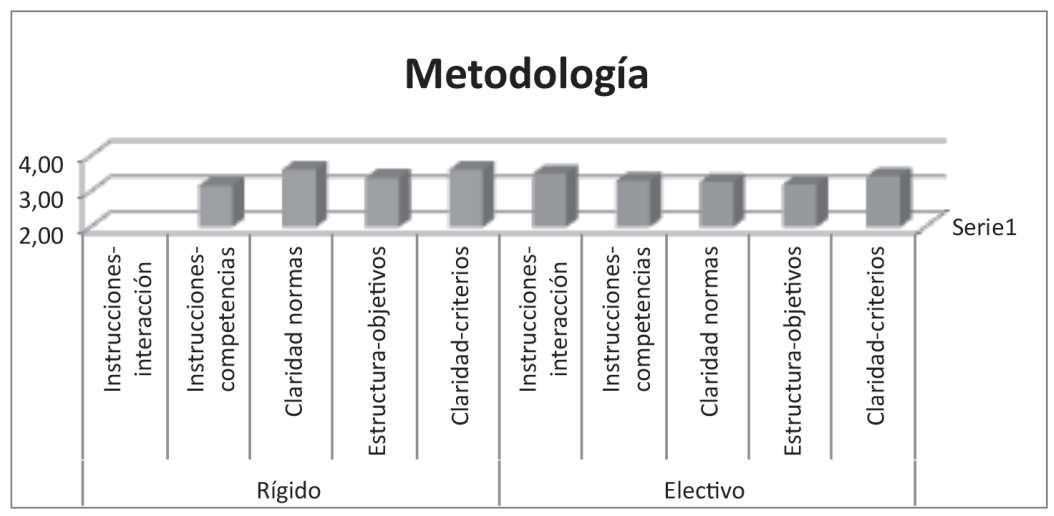

Fuente. Elaboración propia de las autoras.

En el desarrollo de las competencias del curso, el resultado arrojado es $\mathrm{t}=0,721448$ valor-P $=0,474285$, aceptando la hipótesis nula, de ausencia de diferencias significativas entre los dos grupos (Figura 3).

Figura 3. Comparación del desarrollo de competencias en los grupos control (rígido) y experimental (electivo).

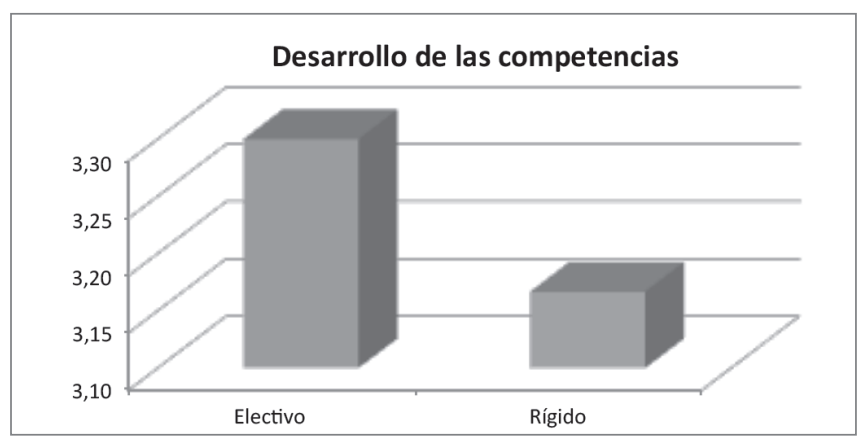

Fuente. Elaboración propia de las autoras.

La variable percepción del aprendizaje, no presentó diferencias significativas en los grupos (Figura 4).

Figura 4. Comparación de la percepción del aprendizaje en los grupos control (rígido) y experimental (electivo).

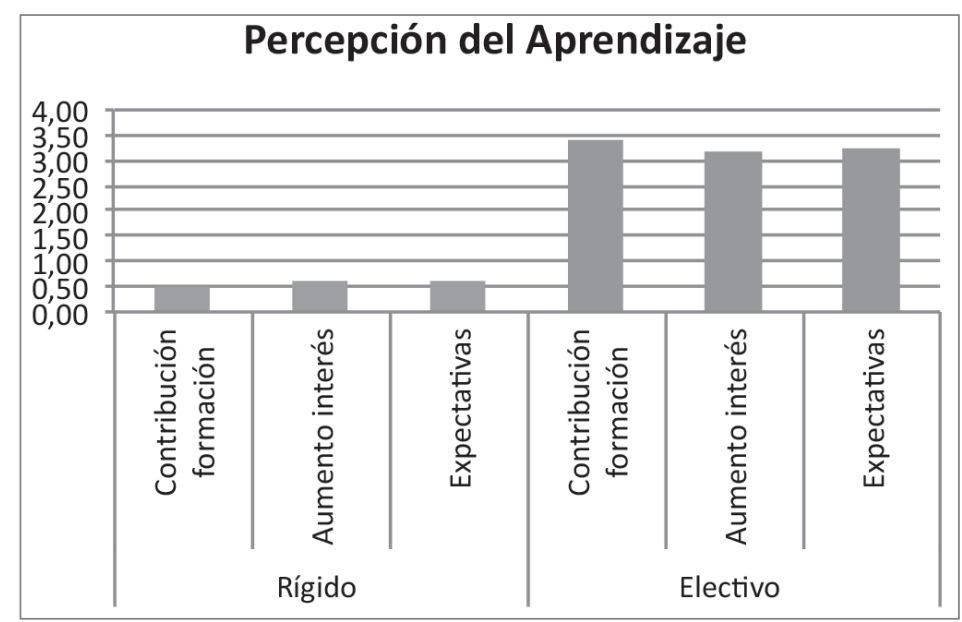

Fuente. Elaboración propia de las autoras. 
En el aumento del conocimiento de los estudiantes sobre los temas abordados, los resultados fueron: $t=-0,566352$ y un $P=0,578555$ de ausencia de diferencias significativas entre los dos grupos (Figura 5).

Figura 5. Comparación de la percepción del aprendizaje en cuanto al aumento de conocimiento en los grupos control (rígido) y experimental (electivo).

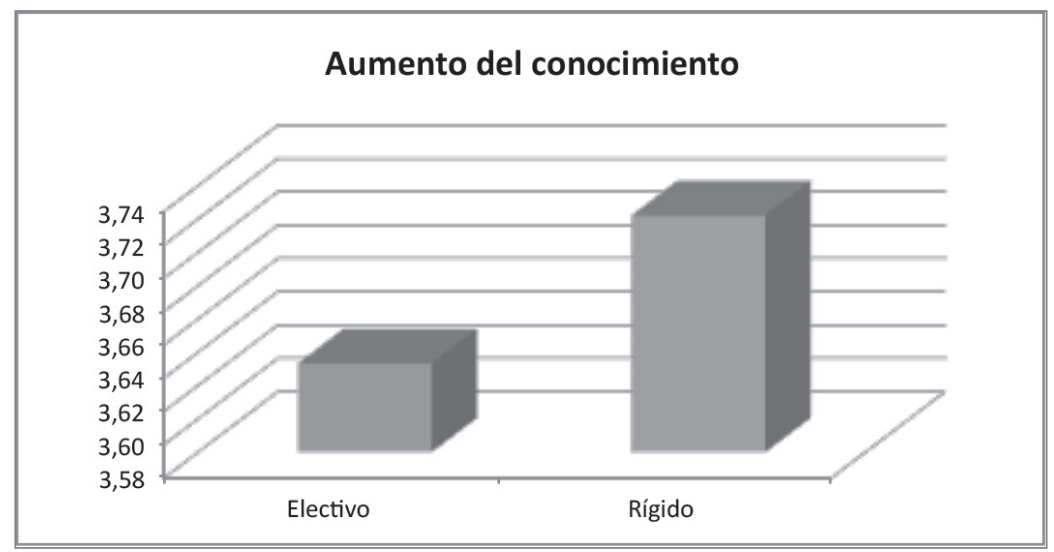

Fuente. Elaboración propia de las autoras.

En la medición que se hizo a los estudiantes a través de la encuesta de satisfacción, se tuvo en cuenta un agregado de indicadores, cada uno con unos componentes particulares que permitieron obrar en la variable. La mayoría de los indicadores no presentó diferencias estadísticamente significativas entre los grupos rígido y electivo. Sin embargo, existe relevancia en el grado en que el tutor del curso integra la teoría y la práctica, donde se encontraron diferencias significativas entre los grupos, con un valor $\mathrm{t}=-2,08847$ y un valor $-\mathrm{P}=0,042444$, siendo superior el puntaje en el grupo experimental (Figura 6).

Figura 6. Comparación tutor del curso y la integración de este con la teoría y la práctica en los grupos control (rígido) y experimental (electivo).

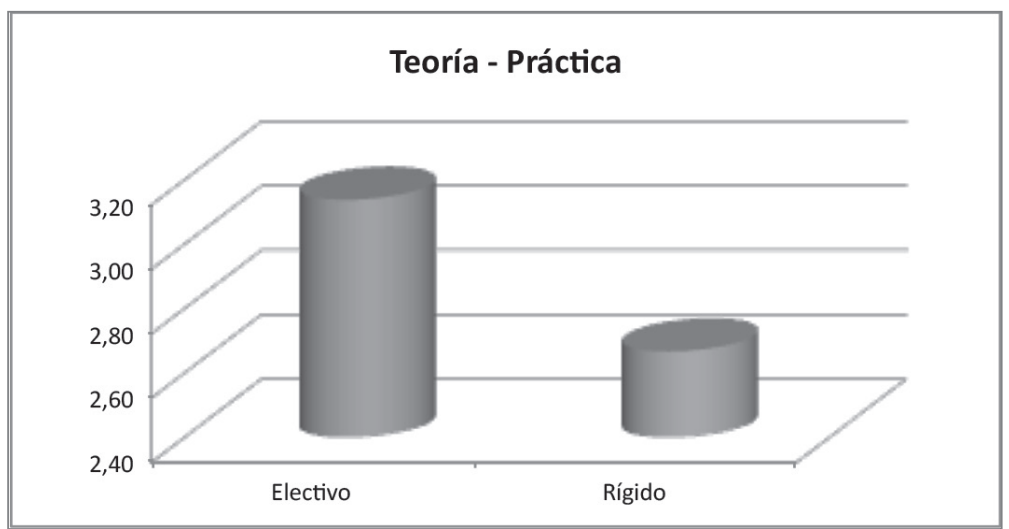

Fuente. Elaboración propia de las autoras.

La variable deserción presentó diferencias significativas en los grupos, se calculó el estadístico $z$ con los siguientes valores: $z$ calculado $=$ 2,36105 valor- $P=0,0182232$. El mayor índice de deserción lo tuvo el aula rígida (grupo control).
Se observa (Figura 7) la comparación entre los grupos, mientras que en los otros casos (Figura 8, Figura 9) se presentan las proporciones de deserción para cada uno de los grupos. 
Figura 7. Comparación de la deserción en los grupos control (rígido) y experimental (electivo).

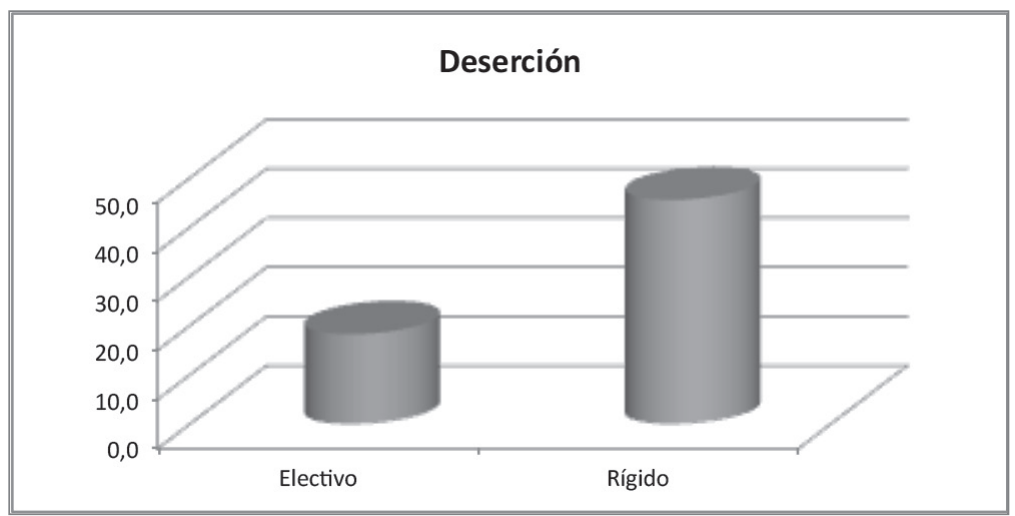

Fuente. Elaboración propia de las autoras.

Figura 8. Deserción grupo control (rígido). En el grupo electivo proporciones muestrales $=0,18$.

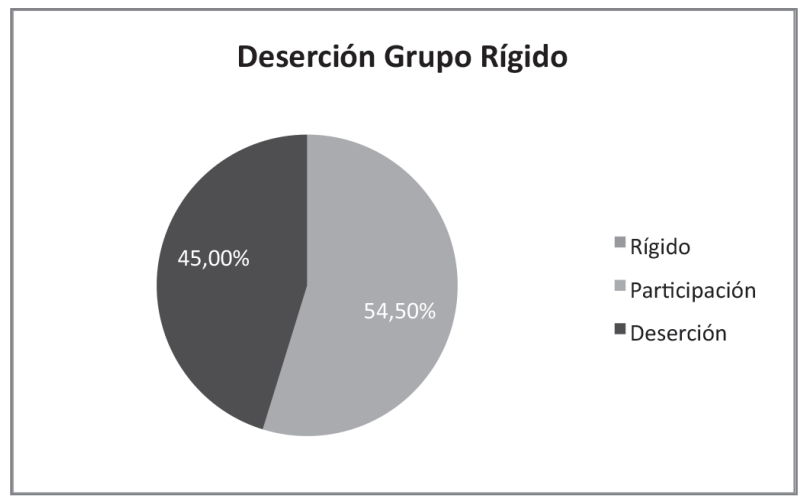

Fuente. Elaboración propia de las autoras.

En cuanto a los niveles de satisfacción, tampoco se evidenciaron diferencias significativas entre los grupos. Los resultados $t=1,15261$; valor-P $=0,255023$.

Figura 10. Satisfacción grupo electivo (Experimental).

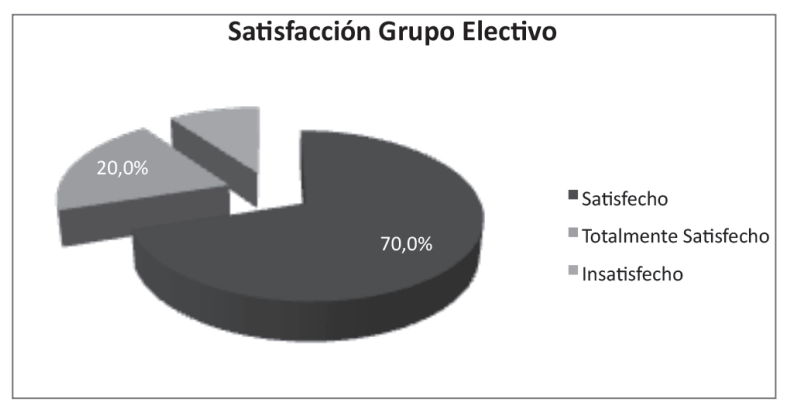

Fuente.Elaboración propia de las autoras.
Figura 9. Deserción grupo electivo (experimental).

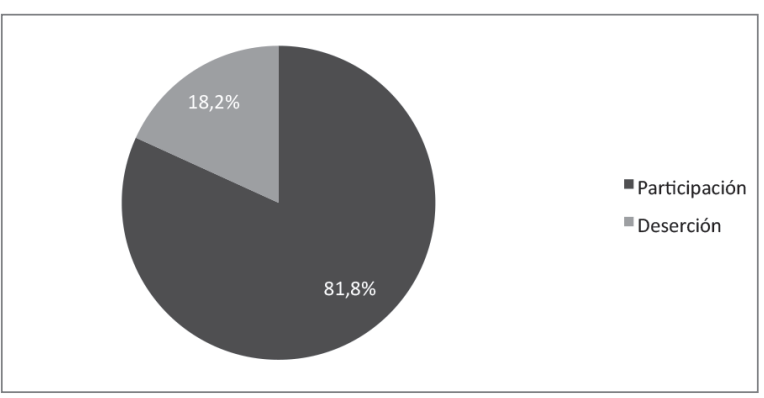

Fuente. Elaboración propia de las autoras.

Figura 11. Satisfacción grupo rígido (Control).

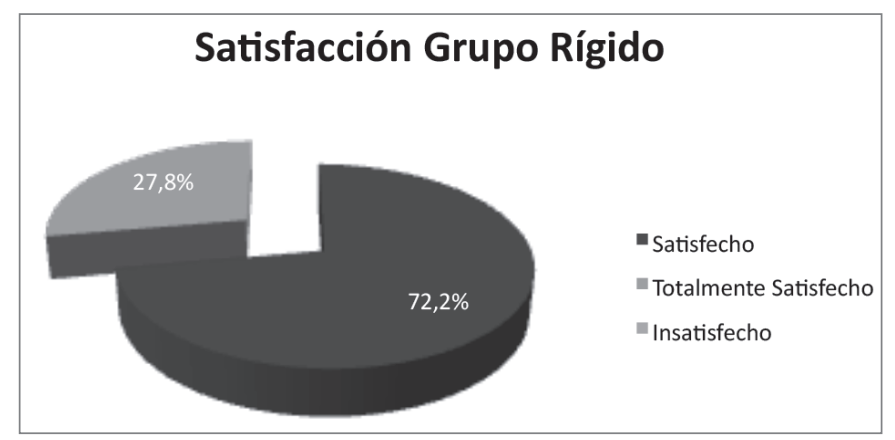

Fuente. Elaboración propia de las autoras. 


\section{DISCUSIÓN}

I analizar los efectos de la electividad
en los cursos temáticos del área socio-
humanística sobre la calidad del aprendizaje en entornos virtuales, los resultados muestran que incluir la electividad no genera efectos en el rendimiento académico de los estudiantes, según (Correa \& Gonçalves, s.f.) para la elaboración y diseño de cursos electivos existen unas fases que se deben desarrollar tales como: la valoración de la instrucción, diseño de la instrucción, producción del curso e implementación. En la fase de valoración de la instrucción es importante p.190 reconocer e identificar actitudes, habilidades y conocimientos previos que tiene el estudiante, lo que propende por un mejor desarrollo del material y claridad en los objetivos del curso, enfocados al desarrollo del pensamiento crítico y significativo centrado en el estudiante. El curso de Negociación Intercultural, contó con el desarrollo de dos aulas una con componente electivo (grupo experimental) y la otra aula rígida (grupo control), basadas en modelo de la Facultad de Estudios en Ambientes Virtuales de la Universidad EAN de desarrollo de competencias.

En cuanto al desarrollo de las competencias, se acepta la hipótesis nula ya que hay ausencia de diferencias significativas en los grupos; el Modelo Educativo de la Universidad EAN, trabaja claramente la flexibilidad dando cumplimiento a elementos como los créditos académicos, las unidades de estudios nucleares, transversales, anfitriones, electivas, el sistema de ciclos académicos, la no existencia de prerrequisitos en los currículos, la pertinencia, la formación integral y el aspecto sistémico; lo que a través del modelo conductual, permite en la modalidad virtual trabajar con un diseño de instrucciones claras y adecuadas para el manejo de las aulas del curso de negociación, las dos aulas cuentan con instrucciones y competencias iguales.

En la medición del nivel de satisfacción de los estudiantes durante el desarrollo del curso de negociación intercultural se evaluaron indicadores como la metodología, la percepción del aprendizaje, el nivel de conocimientos con respecto a las temáticas abordadas y el tutor del curso. En la mayoría de los factores no se encontraron diferencias estadísticamente significativas en los grupos, lo que significa que los efectos en la electividad no generan impactos directos en el aprendizaje, sino que se deben revisar algunos indicadores de manera específica, como la deserción y la integración teóricopráctica del tutor del curso.

En cuanto a la satisfacción, se evidenció mayor grado de satisfacción en el grupo rígido (control), que en el grupo electivo (experimental). De este resultado se puede analizar en qué grado se satisfacen aspectos como la coherencia, el alcance del curso, su pertinencia y aspectos de flexibilidad. En este contexto el estudiante puede generar algunos niveles de incoherencia que se trabajaron en la fase uno de esta investigación, relacionados con el concepto de disonancia cognitiva (Reig, 2012), ya que el estudiante no hace una relación directa con lo que sabe, cree y siente.

Los participantes del curso de Negociación intercultural, en el marco de esta investigación, fueron estudiantes de pregrado de la modalidad virtual, donde se manejan bloques temáticos pero sin una incorporación de electividad en ellos, lo que significa que deben estudiar todas las temáticas asignadas para un bloque, por lo que la inclusión de la electividad en áreas como las humanidades sería un verdadero reto que 
podría facilitar la interacción del estudiante con este tipo de áreas del conocimiento.

La Universidad EAN traza la electividad como un elemento fundamental de la flexibilidad educativa, lo que explica la sinergia directa con el currículo y el modelo de enseñanza. Plantea Canudas (1972) algunos aspectos para la electividad, cómo reconocer las aptitudes individuales de los estudiantes, ofreciendo diferencias de calidad e intensidad, de tipo y jerarquía. La electividad le permite al estudiante la posibilidad de multidisciplinariedad para realizar su propia carta de navegación académica, proceso que les genera algún grado de rechazo debido a la costumbre que tienen de que las temáticas de los cursos e instrucciones sean preestablecidas por el tutor, los estudiantes no deben tomar decisiones sino seguir paso a paso la instrucción para el desarrollo de una guía de trabajo dentro del bloque de estudio, lo que se traduce en un rechazo al cambio (Monereo, 2010) o salirse de la zona de confort.

Con relación al tutor del curso, indicador teórico práctico, se debe hacer claridad en que en el modelo del curso no hubo actividades sincrónicas, por lo cual esta variable presentó deferencias significativas que afectaron la percepción de los participantes en el estudio con respecto a la calidad del curso.

Los hallazgos sobre la variable deserción evidenciaron la existencia de una diferencia estadísticamente significativa entre los grupos. En la fase uno de esta investigación, la deserción en el grupo control también tuvo un resultado significativo con respecto al grupo experimental. El tema de la deserción escolar contiene aspectos socioculturales, económicos, familiares, entre otros, que llevan al estudiante al abandono de su proceso formativo. En el desarrollo del curso de Negociación intercultural, el abandono tiene que ver con el no ingreso al aula, la baja interacción con los contenidos, con los pares académicos y con el tutor; o la no comunicación asincrónica con el tutor para aclarar el desarrollo de la guía de trabajo. En la modalidad virtual la deserción se relaciona directamente con la motivación e incapacidad de asumir el proceso de formación como propio, debido a que el modelo de la FEAV se basa en autoformación, lo que puede ir en contravía con el manejo de otras responsabilidades directas del estudiante en ámbitos como el laboral, personal y familiar.

Es imperioso para las instituciones de educación superior que tienen modalidad virtual, manejar nuevas e innovadoras herramientas para gestionar la deserción, ya que el estudiante no está siempre interactuando de manera sincrónica, sino la mayoría de las veces a través de canales asincrónicos, por ende es de gran importancia el modelo, la apropiación y acción tutorial que se le ofrezca para ser acompañado en su proceso de autoformación. Arranza, Aguado \& Lucia (2008), plantean que los niveles de deserción en la modalidad a distancia son más altos debido, en gran parte, a las propias dificultades del medio, a las características del curso y al interés del alumno (p.7).

Por lo anterior, la motivación es un elemento fundamental en el relacionamiento del estudiante, ya que se debe propender por generar, mantener y manejar la curiosidad del alumno; es importante que se le impongan retos superables, de lo contrario es posible que se sienta frustrado y sobre todo que pierda el interés por aprender (Arranza, Aguado \& Lucia, 2008, p.7).

La modalidad virtual viene en un vertiginoso crecimiento, lo que se presenta como un reto para la Universidad que ha optado por este modelo, pues son varios los factores a tener en cuenta a la hora de hacer oferta educativa en este campo, ya que existen ventajas y desventajas para el estudiante, quien tiene en sus manos su proceso de autoformación y debe generar varios compromisos y asumir las responsabilidades para ello. Igualmente, las universidades deben 
propender por la formación constante de sus docentes en los temas relacionados con la educación virtual y fortalecer las herramientas pedagógicas y didácticas para que la acción tutorial presente mejores resultados en la permanencia de los estudiantes. La fase uno y dos de esta investigación demuestran que la inclusión de nuevos aspectos como la electividad disminuye significativamente la deserción de los estudiantes en entornos virtuales de aprendizaje.

\section{CONCLUSIONES}

$\mathbf{L}$ os hallazgos de esta investigación muestran que, aunque no existen diferencias significativas entre los grupos rígido (control) y electivo (experimental) en la mayoría de las variables dependientes, como producto de la manipulación de la variable independiente, laelectividadsíesunfactorconcluyente para decretar los niveles de deserción y satisfacción de los estudiantes, debido a que el desarrollo del curso de negociación intercultural se realizó de manera voluntaria por estudiantes del área socio-humanística de pregrado de la Facultad de Estudios en Ambientes Virtuales de la Universidad EAN bajo la modalidad virtual y dentro de las premisas que en este modelo la deserción es mucho más alta. Los resultados demuestran, además, los niveles de dificultad en el manejo de las diferentes variables con respecto a la metodología del curso y la percepción de los estudiantes sobre el tutor. En los dos grupos se aprecia una semejanza en el nivel de resultados, así como en los indicadores de rendimiento, aumento del conocimiento y satisfacción, existiendo diferencias significativas únicamente en la relación entre teoría y práctica y el nivel de deserción, siendo indicadores favorables para el grupo experimental.

La revisión teórica de los efectos de la electividad en la calidad del aprendizaje en entornos virtuales, permitió analizar los cambios que han generado las Tecnologías de la Información y la Comunicación (TIC) en las relaciones y ecosistemas educativos actuales, puesto que las estructuras sociales son interdependientes, proceso que se concreta con el manejo y análisis de la diferentes variables de la investigación.

En el modelo de aprendizaje de la Facultad de Estudios en Ambientes Virtuales (FEAV), se presenta claramente el enfoque por competencias y el fundamento en la teoría conductual, ya que se trabaja con instrucciones claras y concretas a través de la creación de Ambientes Virtuales de Aprendizaje (AVAS) y Objetos Virtuales de Aprendizaje.

La electividad en los cursos de negociación intercultural se abordó con la posibilidad de selección de las temáticas y los formatos de presentación de los productos a desarrollar por los estudiantes como parte del ecosistema educativo donde el aprendizaje se centra en el estudiante; además, sin desatender a la enseñanza y el acompañamiento del tutor a través de la acción tutorial. La modalidad virtual permite una clara planeación, organización, alineación y proyección del AVA, que reúne los contenidos disciplinares y específicos del bloque de estudio, Ios OVAS, las actividades, la evaluación y las instrucciones pertinentes para el desarrollo del proceso de formación de los estudiantes y la interacción de estos con el entorno virtual de aprendizaje. 


\section{REFERENCIAS BIBLIOGRÁFICAS}

Arranza, V. Aguado, D. \& Lucia, B. (2008). La influencia del tutor en el seguimiento de programas elearning. Estudio de acciones en un caso práctico. Recuperado de: http://www.redalyc.org/ articulo.oa?id=231316494001

Correa, J \& Gonçalves, M. (S.F.). Diseño y validación de un curso electivo orientado al uso pedagógico de las TIC para la EAD. Recuperdo de: http://www.ucv.ve/fileadmin/user_upload/ vrac/documentos/Curricular_Documentos/Evento/Ponencias_1/Correa_Juana_y_Goncalves_ Mario.pdf

Cunadas, L. (1972). El Currículum de Estudios en la Enseñanza Superior. Revista de Educación Superior 2. Recuperado de: http://publicaciones.anuies.mx/revista/2/1/2/es/el-curriculum-deestudios-en-la-ensenanza-superior

Freire, P. (2005). Pedagogía del oprimido. México: Editorial Siglo XXI.

Hernández, R. Fernández, C. y Baptista, P. (2010). Metodología de la investigación. México: McGraw Hill

Ísola, A., Guerrero, M., Portillo, M., Ferreiros, S., Bursztyn, A. \& Granado, S. (2013). Las asignaturas electivas como eje de la formación del ingeniero ante los desafíos del desarrollo tecnológico. Buenos Aires: Universidad Tecnológica Nacional. Recuperado de: http://conaiisi.unsl.edu. ar/2013/19-501-1-DR.pdf

Moreira, M. (2000). Aprendizaje significativo: Teoría y Práctica. Madrid: Ediciones Visor S.A.

Monereo, C. (2010). iSaquen el libro de texto! Resistencia, obstáculos y alternativas en la formación de los docentes para el cambio educativo. Recuperado de: http://www.revistaeducacion. educacion.es/re352/re352_26.pdf

Ortega, S. \& Moreno, M. (2013). La flexibilidad didáctica en entornos virtuales de aprendizaje. Universidad EAN. Facultad de Estudios en Ambientes Virtuales. Revista Virtualmente, 2(2), 5-23.

Prieto y Van de Pol. (2006). E-Learning comunicación y educación el diálogo continúa en el ciberespacio. RNTC: Hilversum. Recuperado de http://recursostic.javeriana.edu.co/diplo-mado/e_ learning_comunicacion_y_educacion.pdf

Reig, D. (2012). Disonancia cognitiva y apropiación de las TIC. Revista Telos - Revista de Pensamiento sobre Tecnología y Sociedad. 90. Recuperado de: http://sociedadinformacion.fundacion. telefonica.com/DYC/TELOS/REVISTA/TribunasdelaComunicacin_90TELOS_TRIBUNA2/ seccion=1213\&idioma=es_ES\&id $=2012020215200001 \&$ activo $=7$.do

Universidad EAN (2013). Modelo Educativo. Fundamentación. Bogotá: Universidad EAN.

Vila, E. (2011). Racionalidad, diálogo y acción: Habermas y la pedagogía crítica. Revista Iberoamericana de Educación 56/3 - 15/10/11. Organización de Estados Iberoamericanos para la Educación, la Ciencia y la Cultura (OEI-CAEU). Recuperado de http://www.rieoei.org/deloslectores/3652Vila. pdf 